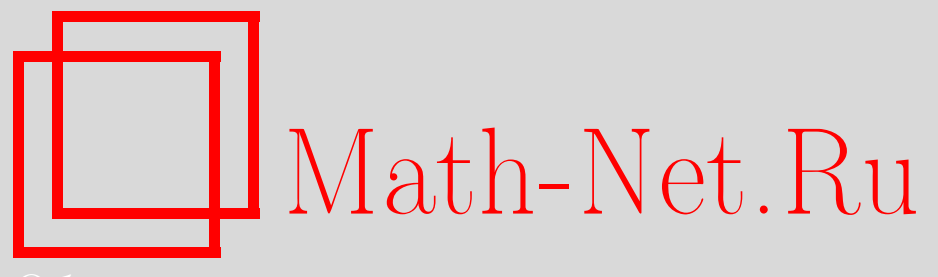

М. А. Кравцов, А. П. Крачковский, О некоторых свойствах трехиндексных транспортных многогранников, Дискрет. матем., 1999, том 11, выпуск 3, 109-125

DOI: https://doi.org/10.4213/dm383

Использование Общероссийского математического портала Math-Net.Ru подразумевает, что вы прочитали и согласны с пользовательским соглашением http: //www . mathnet.ru/rus/agreement

Параметры загрузки:

IP : 54.162 .85 .209

26 апреля 2023 г., $18: 26: 36$ 


\title{
О некоторых свойствах трехиндексных транспортных многогранников
}

\author{
(C) 1999 г. М. А. Кравцов, А. П. Крачковский
}

Для всякого трехиндексного планарного транспортного многогранника (3ПТМ) $M$ порядка $m \times n \times k$ размерности $d$ указан трехиндексный аксиальный транспортный многогранник (3-ATM) $M^{\prime}$ порядка $m k \times n k \times m n$, у которого найдена $d$-грань, комбинаторно эквивалентная многограннику $M$, и наоборот, для всякого 3-АТМ $M$ порядка $m \times n \times k$ указан 3-ПТМ $M^{\prime}$ порядка $(m+1) \times(n+1) \times(k+1)$, у которого найдена $(m n k-m-n-k+2)$-грань комбинаторно эквивалентная многограннику $M$. С помощью этих результатов получен ряд новых свойств, касающихся строения трехиндексных транспортных многогранников.

Работа выполнена при поддержке Фонда фундаментальных исследований Республики Беларусь, проект Ф95-70.

\section{1. Введение}

Исследованию области определения $p$-индексных транспортных задач линейного программирования, имеющих большую сферу приложений в математической кибернетике, экономике и теории расписаний, посвящен ряд работ (см., например, [1-6]). Однако наиболее существенные и глубокие результаты получены лишь для случая, когда $p=2[1,3,6-11]$.

В отличие от классической (двухиндексной) транспортной задачи, у которой матрица ограничений всегда унимодулярна, матрица ограничений многоиндексной транспортной задачи линейного программирования не является унимодулярной. Поэтому многоиндексный транспортный многогранник может иметь и нецелочисленные вершины при целочисленных исходных данных. Это, естественно, затрудняет нахождение целочисленных решений, превращая, например, проблему проверки целочисленной совместности трехиндексной планарной транспортной задачи в NPполную [12]. Установлена также NP-полнота аксиальной и планарной трехиндексной проблемы выбора $[13,14]$. Этим, в частности, обьясняется большой интерес к свойствам и методам решения многоиндексных транспортных задач, который оправдывает неослабевающие усилия по исследованию структуры многогранников этого класса задач.

Настоящая работа посвящена исследованию полиэдральных аспектов трехиндексных планарных и аксиальных транспортных задач, то есть вопросов, связанных со строением областей определения таких задач. Найдены также оценки снизу 
для максимального диаметра, радиуса, числа вершин и целочисленных вершин 3ПТМ заданного порядка. Показано, что в классе 3-ПТМ порядка $m \times n \times k, m \geqslant 6$, $n \geqslant 4, k \geqslant 3$, определенных целочисленными матрицами, имеются многогранники, у которых нет ни одной целочисленной точки.

Сначала введем некоторые определения и обозначения, которыми будем пользоваться далее.

Трехиндексным планарным транспортным многогранником (3-ПТМ) порядка $m \times n \times k, m, n, k>1$, называется область определения трехиндексной планарной транспортной задачи [1], то есть множество $M(A, B, C)$ матриц $x=\left\|x_{i j t}\right\|_{m \times n \times k}$, элементы которых удовлетворяют следующим условиям:

$$
\begin{aligned}
\sum_{j=1}^{n} x_{i j t}=a_{i t} & \forall(i, t) \in N_{m} \times N_{k}, \\
\sum_{i=1}^{m} x_{i j t}=b_{j t} & \forall(j, t) \in N_{n} \times N_{k}, \\
\sum_{t=1}^{k} x_{i j t}=c_{i j} & \forall(i, j) \in N_{m} \times N_{n}, \\
x_{i j t} \geqslant 0 & \forall(i, j, t) \in N_{m} \times N_{n} \times N_{k},
\end{aligned}
$$

где

$$
A=\left\|a_{i t}\right\|_{m \times k}, \quad B=\left\|b_{j t}\right\|_{n \times k}, \quad C=\left\|c_{i j}\right\|_{m \times n}
$$

- матрицы с неотрицательными элементами, $N_{p}=\{1,2, \ldots, p\}$.

Наряду с $M(A, B, C)$ будем рассматривать трехиндексный аксиальный транспортный многогранник (3-АТМ) $M(a, b, c)$ порядка $m \times n \times k, m, n, k>1$, представляющий собой множество матриц $x=\left\|x_{i j t}\right\|_{m \times n \times k}$, элементы которых удовлетворяют условиям

$$
\begin{aligned}
\sum_{j=1}^{n} \sum_{t=1}^{k} x_{i j t} & =a_{i} \quad \forall i \in N_{m}, \\
\sum_{i=1}^{m} \sum_{t=1}^{k} x_{i j t} & =b_{j} \quad \forall j \in N_{n}, \\
\sum_{i=1}^{m} \sum_{j=1}^{n} x_{i j t} & =c_{t} \quad \forall t \in N_{k}, \\
x_{i j t} \geqslant 0 & \forall(i, j, t) \in N_{m} \times N_{n} \times N_{k},
\end{aligned}
$$

где

$$
a=\left(a_{1}, a_{2}, \ldots, a_{m}\right), \quad b=\left(b_{1}, b_{2}, \ldots, b_{n}\right), \quad c=\left(c_{1}, c_{2}, \ldots, c_{k}\right)
$$

- векторы с действительными положительными компонентами,

$$
\sum_{i=1}^{m} a_{i}=\sum_{j=1}^{n} b_{j}=\sum_{t=1}^{k} c_{t} .
$$

Неопределяемые здесь термины и обозначения взяты из [1]. 


\section{2. Аксиальное представление 3-ПТМ}

В этом разделе для всякого 3-ПТМ $M(A, B, C)$ порядка $m \times n \times k$ размерности $d$ указан 3-АTM $M\left(a^{\prime}, b^{\prime}, c^{\prime}\right)$ порядка $m k \times n k \times m n$, у которого найдена $d$-грань комбинаторно эквивалентная многограннику $M(A, B, C)$. Такую $d$-грань будем называть аксиальным представлением 3-ПТМ $M(A, B, C)$. Здесь компоненты векторов

$$
a^{\prime}=\left(a_{1}^{\prime}, a_{2}^{\prime}, \ldots, a_{m k}^{\prime}\right), \quad b^{\prime}=\left(b_{1}^{\prime}, b_{2}^{\prime}, \ldots, b_{n k}^{\prime}\right), \quad c^{\prime}=\left(c_{1}^{\prime}, c_{2}^{\prime}, \ldots, c_{m n}^{\prime}\right)
$$

определяются однозначно по элементам матриц $A, B$ и $C$ соответственно.

В самом деле, каждой паре $(i, t)$ из множества $N_{m} \times N_{k}$ поставим в соответствие индекс $i_{1} \in N_{m k}$ согласно формуле

$$
i_{1}=(i-1) k+t .
$$

Очевидно, что это соответствие взаимно однозначное. Действительно, для каждого индекса $i_{1} \in N_{m k}$ соответствующая пара $(i, t) \in N_{m} \times N_{k}$ обозначается $i_{1} \rightarrow(i, t)$ и определяется по формулам

$$
i=] i_{1} / k\left[, \quad t=i_{1}-(i-1) k,\right.
$$

где $] h[$ - наименьшее целое число, не меньшее числа $h$.

Аналогично, парам $(j, t) \in N_{n} \times N_{k}$ и $(i, j) \in N_{m} \times N_{n}$ поставим в соответствие индексы $i_{2} \in N_{n k}$ и $i_{3} \in N_{m n}$, определяемые по формулам

$$
i_{2}=(j-1) k+t, \quad i_{3}=(i-1) n+j .
$$

Теперь по заданным матрицам $A, B$ и $C$ единственным образом определяются векторы $a^{\prime}, b^{\prime}$ и $c^{\prime}$ с помощью формул

$$
\begin{aligned}
& a_{i_{1}}^{\prime}=a_{i t} \Longleftrightarrow i_{1} \rightarrow(i, t) \quad \forall i_{1} \in N_{m k}, \\
& b_{i_{2}}^{\prime}=b_{j t} \Longleftrightarrow i_{2} \rightarrow(j, t) \quad \forall i_{2} \in N_{n k}, \\
& c_{i_{3}}^{\prime}=c_{i j} \Longleftrightarrow i_{3} \rightarrow(i, j) \quad \forall i_{3} \in N_{m n} .
\end{aligned}
$$

Введем обозначение

$$
Q=N_{m k} \times N_{n k} \times N_{m n} .
$$

С помощью формул (1) и (2), определим отображение

$$
\gamma: N_{m} \times N_{n} \times N_{k} \rightarrow Q,
$$

которое каждой тройке $(i, j, t) \in N_{m} \times N_{n} \times N_{k}$ ставит в соответствие некоторую тройку $\left(i_{1}, i_{2}, i_{3}\right) \in Q$. Обозначим через $E$ множество значений отображения $\gamma$, т.е.

$$
E=\left\{\left(i_{1}, i_{2}, i_{3}\right) \in Q:\left(i_{1}, i_{2}, i_{3}\right)=\gamma(i, j, t) \forall(i, j, t) \in N_{m} \times N_{n} \times N_{k}\right\} .
$$

Лемма 1. Отображение $\gamma: N_{m} \times N_{n} \times N_{k} \rightarrow E$ является взаимно однозначным.

Доказательство. Очевидно, что каждой тройке $(i, j, t) \in N_{m} \times N_{n} \times N_{k}$ соответствует единственная тройка $\left(i_{1}, i_{2}, i_{3}\right) \in E$. Теперь покажем обратное, то есть покажем, что любой тройке множества $E$ соответствует единственная тройка множества 
$N_{m} \times N_{n} \times N_{k}$. Зафиксируем некоторую тройку $\left(i_{1}^{0}, i_{2}^{0}, i_{3}^{0}\right) \in E$ и рассмотрим систему уравнений относительно неизвестных $i, j, t$

$$
\begin{aligned}
& k i+t=i_{1}^{0}+k, \\
& k j+t=i_{2}^{0}+k, \\
& n i+j=i_{3}^{0}+n .
\end{aligned}
$$

Определитель этой системы равен $-k(n+1)$. Следовательно, система (6) имеет единственное решение $\left(i^{0}, j^{0}, t^{0}\right)$, которое в силу определения множества $E$ удовлетворяет условиям

$$
i^{0} \in N_{m}, \quad j^{0} \in N_{n}, \quad t^{0} \in N_{k} .
$$

Лемма 1 доказана.

Положим $H=Q \backslash E$. Заметим, что множество $H$ определяется однозначно в силу однозначности определения множества $E$, задаваемого формулами (1) и (2).

Введем в рассмотрение множество

$$
M_{H}\left(a^{\prime}, b^{\prime}, c^{\prime}\right)=\left\{y=\left\|y_{i_{1} i_{2} i_{3}}\right\| \in M\left(a^{\prime}, b^{\prime}, c^{\prime}\right): y_{i_{1} i_{2} i_{3}}=0 \forall\left(i_{1}, i_{2}, i_{3}\right) \in H\right\} .
$$

Легко видеть, что это множество является некоторой гранью 3-АТМ, порядок которой равен $m k \times n k \times m n$.

Лемма 2. Справедливо соотношение

$$
M(A, B, C) \neq \varnothing \Longleftrightarrow M_{H}\left(a^{\prime}, b^{\prime}, c^{\prime}\right) \neq \varnothing .
$$

Доказательство. Докажем необходимость. Пусть $x$ - некоторая матрица 3-ПТМ $M(A, B, C)$. Используя лемму 1 , по матрице $x$ построим матрицу $y$ согласно формуле

$$
y_{i_{1} i_{2} i_{3}}= \begin{cases}x_{i j t}, & \text { если }\left(i_{1}, i_{2}, i_{3}\right) \in E, \quad\left(i_{1}, i_{2}, i_{3}\right)=\gamma(i, j, t), \\ 0, & \text { если }\left(i_{1}, i_{2}, i_{3}\right) \in H .\end{cases}
$$

Легко проверить, что $y \in M_{H}\left(a^{\prime}, b^{\prime}, c^{\prime}\right)$.

Докажем достаточность. Пусть $y$ - некоторая матрица из множества $M_{H}\left(a^{\prime}, b^{\prime}, c^{\prime}\right)$. $\mathrm{C}$ помощью леммы 1 по матрице $y$ построим матрицу $x$ согласно формуле

$$
x_{i j t}=y_{i_{1} i_{2} i_{3}}, \quad\left(i_{1}, i_{2}, i_{3}\right)=\gamma(i, j, t) \quad \forall(i, j, t) \in N_{m} \times N_{n} \times N_{k} .
$$

Нетрудно проверить, что $x \in M(A, B, C)$. Лемма 2 доказана.

В дальнейшем будем предполагать, что 3 -ПТМ $M(A, B, C) \neq \varnothing$. Тогда в силу леммы 2 многогранник $M_{H}\left(a^{\prime}, b^{\prime}, c^{\prime}\right) \neq \varnothing$.

Лемма 3. Справедливо равенство

$$
\operatorname{dim} M(A, B, C)=\operatorname{dim} M_{H}\left(a^{\prime}, b^{\prime}, c^{\prime}\right) .
$$

Доказательство. Известно [1], что выпуклый многогранник $M$ имеет размерность $d$ тогда и только тогда, когда $M$ содержит подмножество, состоящее из $d+1$ аффинно независимых точек, а любое подмножество $S \subset M$, состоящее из $d+2$ точек аффинно зависимо. Размерность пустого множества считается равной -1 . 
Пусть $\operatorname{dim} M(A, B, C)=d$, a $\left\{x^{1}, x^{2}, \ldots, x^{d+1}\right\}-$ аффинно независимое множество точек (матриц) многогранника $M(A, B, C)$. Тогда по формуле (7) определим множество $\left\{y^{1}, y^{2}, \ldots, y^{d+1}\right\}$ точек (матриц) многогранника $M_{H}\left(a^{\prime}, b^{\prime}, c^{\prime}\right)$. Легко видеть, что из аффинной независимости множества $\left\{x^{1}, x^{2}, \ldots, x^{d+1}\right\}$ следует аффинная независимость множества $\left\{y^{1}, y^{2}, \ldots, y^{d+1}\right\}$. Поэтому

$$
\operatorname{dim} M_{H}\left(a^{\prime}, b^{\prime}, c^{\prime}\right) \geqslant d .
$$

Предположим, что $\operatorname{dim} M_{H}\left(a^{\prime}, b^{\prime}, c^{\prime}\right)>d$. Это означает, что найдется подмножество

$$
\left\{\hat{y}^{1}, \hat{y}^{2}, \ldots, \hat{y}^{d+2}\right\} \subset M_{H}\left(a^{\prime}, b^{\prime}, c^{\prime}\right),
$$

состоящее из $d+2$ аффинно независимых точек. Тогда по формуле (8) определим множество точек

$$
\left\{\hat{x}^{1}, \hat{x}^{2}, \ldots, \hat{x}^{d+2}\right\} \subset M(A, B, C),
$$

которое также является аффинно независимым. Но это противоречит условию, что $\operatorname{dim} M(A, B, C)=d$. Следовательно, $\operatorname{dim} M_{H}\left(a^{\prime}, b^{\prime}, c^{\prime}\right)=d$. Лемма 3 доказана.

Известно (см. [1]), что размерность всякого непустого 3-ПТМ $M(A, B, C)$ порядка $m \times n \times k$ удовлетворяет неравенствам

$$
0 \leqslant \operatorname{dim} M(A, B, C) \leqslant(m-1)(n-1)(k-1),
$$

причем нижняя и верхняя оценки являются достижимыми. В [15] показано, что любое число вида

$$
(m-1)(n-1)(k-1)-m_{1} n_{1} k_{1}-m_{2} n_{2} k_{2}+1
$$

где

$$
m_{1}+m_{2}=m, \quad n_{1}+n_{2}=n, \quad k_{1}+k_{2}=k
$$

и $m_{1}, m_{2}, n_{1}, n_{2}, k_{1}, k_{2}$ - целые неотрицательные числа, реализуется как размерность некоторого 3-ПТМ порядка $m \times n \times k$.

Напомним [1], что грань $F$ многогранника $M(A, B, C)$ определяется следующим образом:

$$
F=\left\{x \in M(A, B, C): x_{i j t}=0 \forall(i, j, t) \in P\right\},
$$

где $P$ - некоторое подмножество множества $N_{m} \times N_{n} \times N_{k}$. Если $\operatorname{dim} F=s$, то $F$ называется $s$-гранью многогранника $M(A, B, C)$, а 0-грани называются вершинами многогранника $M(A, B, C)$, множество всех вершин обозначаем через $\operatorname{vert} M(A, B, C)$.

Аналогично задается грань многогранника $M_{H}\left(a^{\prime}, b^{\prime}, c^{\prime}\right)$.

Пусть задано отображение

$$
\psi: M(A, B, C) \rightarrow M_{H}\left(a^{\prime}, b^{\prime}, c^{\prime}\right),
$$

которое каждой матрице $x \in M(A, B, C)$ по формуле (7) ставит в соответствие матрицу $y \in M_{H}\left(a^{\prime}, b^{\prime}, c^{\prime}\right)$.

Теорема 1. Отображение $\psi: M(A, B, C) \rightarrow M_{H}\left(a^{\prime}, b^{\prime}, c^{\prime}\right)$ обладает следующими свойствами: 
(1) $\psi$ - взаимно однозначное отображение, имеющее обратное отображение

$$
\psi^{-1}: M_{H}\left(a^{\prime}, b^{\prime}, c^{\prime}\right) \rightarrow M(A, B, C),
$$

определяемое по формуле (8);

(2) точка $x$ является верииной многогранника $M(A, B, C)$ тогда и толъко тогда, когда $\psi(x)$ - вершина многогранника $M_{H}\left(a^{\prime}, b^{\prime}, c^{\prime}\right)$;

(3) множество $F$ является гранъю многогранника $M(A, B, C)$ тогда и толъко тогда, когда $\psi(F)$ - гранъ многогранника $M_{H}\left(a^{\prime}, b^{\prime}, c^{\prime}\right)$.

Доказательство. Справедливость первого свойства непосредственно следует из формул (7) и (8).

Установим справедливость второго свойства. Сначала докажем импликацию

$$
x \in \operatorname{vert} M(A, B, C) \Rightarrow \psi(x) \in \operatorname{vert} M_{H}\left(a^{\prime}, b^{\prime}, c^{\prime}\right) .
$$

Допустим, что для некоторой вершины $x^{0} \in \operatorname{vert} M(A, B, C)$ ее образ $\psi\left(x^{0}\right)$ не является вершиной многогранника $M_{H}\left(a^{\prime}, b^{\prime}, c^{\prime}\right)$. Тогда точка $\psi\left(x^{0}\right)$ представима в виде выпуклой комбинации некоторых вершин $y^{1}, y^{2}, \ldots, y^{r}$ многогранника $M_{H}\left(a^{\prime}, b^{\prime}, c^{\prime}\right)$, то есть

$$
\psi\left(x^{0}\right)=\sum_{i=1}^{r} \lambda_{i} y^{i}
$$

где

$$
r \geqslant 2, \quad \sum_{i=1}^{r} \lambda_{i}=1, \quad \lambda_{i}>0 \quad \forall i \in N_{r} .
$$

Отсюда, учитывая свойство 1 теоремы, получаем, что в многограннике $M(A, B, C)$ существует последовательность вершин

$$
x^{i}=\psi^{-1}\left(y^{i}\right) \quad \forall i \in N_{r}
$$

для которой справедливо равенство

$$
x^{0}=\sum_{i=1}^{r} \lambda_{i} x^{i} .
$$

Но это противоречит предположению, что $x^{0} \in \operatorname{vert} M(A, B, C)$. Следовательно, $\psi\left(x^{0}\right) \in \operatorname{vert} M_{H}\left(a^{\prime}, b^{\prime}, c^{\prime}\right)$.

Аналогично доказывается обратная импликация

$$
\psi(x) \in \operatorname{vert} M_{H}\left(a^{\prime}, b^{\prime}, c^{\prime}\right) \Rightarrow x \in \operatorname{vert} M(A, B, C) .
$$

Таким образом свойство 2 доказано.

Теперь докажем третье свойство. На основании только что доказанного свойства 2 теоремы достаточно доказать свойство 3 для $d$-граней, $d \geqslant 1$, многогранников $M(A, B, C)$ и $M_{H}\left(a^{\prime}, b^{\prime}, c^{\prime}\right)$. Сначала докажем, что если $F$ - грань многогранника $M(A, B, C)$, то $\psi(F)$ - грань многогранника $M_{H}\left(a^{\prime}, t^{\prime}, c^{\prime}\right)$.

Предположим, что для некоторой грани $F^{0}$ многогранника $M(A, B, C)$ множество $\psi\left(F^{0}\right)$, принадлежащее многограннику $M_{H}\left(a^{\prime}, b^{\prime}, c^{\prime}\right)$, не является гранью. Тогда 
в множестве $\psi\left(F^{0}\right)$ найдется матрица $y^{0}$, а в множестве vert $M_{H}\left(a^{\prime}, b^{\prime}, c^{\prime}\right)$ последовательность вершин

$$
y^{i}, \quad i=1,2, \ldots, l, \quad l \geqslant 2, \quad\left\{y^{1}, y^{2}, \ldots, y^{l}\right\} \backslash \operatorname{vert} M_{H}\left(a^{\prime}, b^{\prime}, c^{\prime}\right) \cap \psi\left(F^{0}\right) \neq \varnothing
$$

такие, что справедливо представление

$$
y^{0}=\sum_{i=1}^{l} \lambda_{i} y^{i}
$$

где

$$
\sum_{i=1}^{l} \lambda_{i}=1, \quad \lambda_{i}>0 \quad \forall i \in N_{l} .
$$

Отсюда в силу свойств 1 и 2 теоремы получаем, что в грани $F^{0}$ есть матрица $x^{0}=$ $\psi^{-1}\left(y^{0}\right)$, а в множестве vert $M(A, B, C)$ вершины

$$
x^{i}=\psi^{-1}\left(y^{i}\right) \quad \forall i \in N_{l}, \quad\left\{x^{1}, x^{2}, \ldots, x^{l}\right\} \backslash \operatorname{vert} F^{0} \neq \varnothing,
$$

для которых выполняется равенство

$$
x^{0}=\sum_{i=1}^{l} \lambda_{i} x^{i}
$$

Но это противоречит предположению, что $F^{0}$ - грань многогранника $M(A, B, C)$. Следовательно, $\psi\left(F^{0}\right)$ - грань многогранника $M_{H}\left(a^{\prime}, b^{\prime}, c^{\prime}\right)$.

Аналогично доказывается, что если $\psi(F)$ - грань многогранника $M_{H}\left(a^{\prime}, b^{\prime}, c^{\prime}\right)$, то $F$ - грань многогранника $M(A, B, C)$. Теорема 1 доказана.

Из теоремы 1 вытекает следующее утверждение.

Следствие 1. Многогранник $M_{H}\left(a^{\prime}, b^{\prime}, c^{\prime}\right)$ порядка $m k \times n k \times m n$ всегда является вырожденным.

Напомним $[1,16]$, что два многогранника $M$ и $M^{\prime}$ называются комбинаторно эквивалентными (обозначается $M \cong M^{\prime}$ ), если между множествами их граней всех размерностей существует взаимно однозначное отображение $\varphi$, сохраняющее операцию включения, то есть

$$
F_{1} \subset F_{2} \Longleftrightarrow\left(F_{1}\right) \subset\left(F_{2}\right)
$$

для любых множеств граней $F_{1}, F_{2}$ многогранника $M$.

Теорема 2. Справедливо соотношение

$$
M(A, B, C) \cong M_{H}\left(a^{\prime}, b^{\prime}, c^{\prime}\right)
$$

Важность этой теоремы состоит, в частности, в том, что задача нахождения экстремума линейной функции на 3-ПТМ $M(A, B, C)$ порядка $m \times n \times k$ может быть сведена к решению аналогичной задачи на многограннике $M_{H}\left(a^{\prime}, b^{\prime}, c^{\prime}\right)$ порядка $m k \times n k \times m n($ см. [2]). 
Доказательство. Определим отображение $\varphi$ между множествами граней всех размерностей многогранников $M(A, B, C)$ и $M_{H}\left(a^{\prime}, b^{\prime}, c^{\prime}\right)$, ставя в соответствие каждой грани $F$ многогранника $M(A, B, C)$ грань $\varphi(F)$ многогранника $M_{H}\left(a^{\prime}, b^{\prime}, c^{\prime}\right)$, совпадающую с образом грани $F$ при отображении $\psi$ (см. свойство 3 теоремы 1$)$.

На основании теоремы 1 отображение $\varphi$ является взаимно однозначным. Более того, из свойства 2 теоремы 1 следует, что для любых двух различных граней $F_{1}$ и $F_{2}$ многогранника $M(A, B, C)$ справедливо утверждение

$$
\operatorname{vert} F_{1} \subset \operatorname{vert} F_{2} \Longleftrightarrow \operatorname{vert} \psi\left(F_{1}\right) \subset \operatorname{vert} \psi\left(F_{2}\right)
$$

Отсюда, учитывая, что любая грань многогранника есть выпуклая оболочка своих вершин, заключаем, что отображение $\varphi$ сохраняет операцию включения.

Теорема 2 доказана.

Поскольку множество $M_{H}\left(a^{\prime}, b^{\prime}, c^{\prime}\right)$ представляет собой $d$-грань 3-АТМ $M\left(a^{\prime}, b^{\prime}, c^{\prime}\right)$, где $d=\operatorname{dim} M(A, B, C)$ (см. лемму 3 ), на основании теоремы $2 M_{H}\left(a^{\prime}, b^{\prime}, c^{\prime}\right)$ - аксиальное представление 3-ПТМ $M(A, B, C)$.

Из теоремы 2 получаем следующее утверждение.

Следствие 2. Граф 3-ПТМ $M(A, B, C)$ порядка $m \times n \times k$ изоморфен графу многогранника $M_{H}\left(a^{\prime}, b^{\prime}, c^{\prime}\right)$ порядка $m k \times n k \times m n$.

С помощью теоремы 2 легко устанавливаются следующие свойства, касающиеся комбинаторно эквивалентных многогранников $M(A, B, C)$ и $M_{H}\left(a^{\prime}, b^{\prime}, c^{\prime}\right)$ :

(1) $\operatorname{dim} F=\operatorname{dim} \varphi(F)$ и $F \cong \varphi(F)$;

(2) $f_{s}(M(A, B, C))=f_{s}\left(M_{H}\left(a^{\prime}, b^{\prime}, c^{\prime}\right)\right), s=0,1,2, \ldots, d-1$, где $d=\operatorname{dim} M(A, B, C)$;

(3) $\operatorname{diam} M(A, B, C)=\operatorname{diam} M_{H}\left(a^{\prime}, b^{\prime}, c^{\prime}\right)$;

(4) $r(M(A, B, C))=r\left(M_{H}\left(a^{\prime}, b^{\prime}, c^{\prime}\right)\right)$.

Здесь $f_{s}(M), \operatorname{diam} M$ и $r(M)$ обозначают соответственно число $s$-граней, диаметр и радиус многогранника $M$.

Известно [1], что среди 3-ПТМ порядка $m \times n \times k$ существуют $(m-1)(n-1)(k-1)$ симплексы. Поэтому из теоремы 2 получаем следующее утверждение.

Следствие 3. Среди 3-ATM порядка $m k \times n k \times m n$ существуют многогранники, $y$ которых найдется $(m-1)(n-1)(k-1)$-грань, являющееся симплексом.

\section{3. Планарное представление 3-АТМ}

В данном разделе для всякого 3-АТМ $M(a, b, c)$ порядка $m \times n \times k$ указан 3-ПТМ $M\left(A^{\prime}, B^{\prime}, C^{\prime}\right)$ порядка $(m+1) \times(n+1) \times(k+1)$, у которого найдена $(m n k-m-n-k+2)$ - 
грань комбинаторно эквивалентная многограннику $M(a, b, c)$, где

$$
\begin{aligned}
A^{\prime} & =\left[\begin{array}{ccccc}
R & R & \ldots & R & n R-a_{1} \\
R & R & \ldots & R & n R-a_{2} \\
R & R & \ldots & & \\
R & R & R & n R-a_{m} \\
n R-c_{1} & n R-c_{2} & \ldots & n R-c_{k} & R
\end{array}\right], \\
B^{\prime} & =\left[\begin{array}{ccccc}
R & R & \ldots & R & m R-b_{1} \\
R & R & \ldots & R & m R-b_{2} \\
R & R & \ldots & & \\
m R-c_{1} & m R-c_{2} & \ldots & m R-c_{k} & R
\end{array}\right], \\
C^{\prime} & =\left[\begin{array}{ccccc}
R & R & \ldots & R & k R-a_{1} \\
R & R & \ldots & R & k R-a_{2} \\
R & R & \ldots & R & k R-a_{m} \\
k R-b_{1} & k R-b_{2} & \ldots & k R-b_{n} & R
\end{array}\right], \\
R & =\max \left\{a_{1}, a_{2}, \ldots, a_{m} ; b_{1}, b_{2}, \ldots, b_{n} ; c_{1}, c_{2}, \ldots, c_{k}\right\} .
\end{aligned}
$$

Такую $(m n k-m-n-k+2)$-грань будем называть планарным представлением 3-ATM $M(a, b, c)$.

Имеется глубокая аналогия между материалами настоящего и предыдущего разделов. Поэтому однотипные доказательства будем опускать, уделив больше внимания специфическим особенностям.

Введем обозначение

$G=\left\{(m+1, n+1, t): t \in N_{k}\right\} \cup\left\{(m+1, j, k+1): j \in N_{n}\right\} \cup\left\{(i, n+1, k+1): i \in N_{m}\right\}$.

Очевидно, что непустое множество

$M_{G}\left(A^{\prime}, B^{\prime}, C^{\prime}\right)=\left\{y=\left\|y_{i j t}\right\| \in M\left(A^{\prime}, B^{\prime}, C^{\prime}\right): y_{i j t}=0 \forall(i, j, t) \in G, y_{m+1, n+1, k+1}=R\right\}$ образует некоторую грань многогранника $M\left(A^{\prime}, B^{\prime}, C^{\prime}\right)$.

Лемма 4. Справедливо соотночение

$$
M_{G}\left(A^{\prime}, B^{\prime}, C^{\prime}\right) \neq \varnothing \Longleftrightarrow \sum_{i=1}^{m} a_{i}=\sum_{j=1}^{n} b_{j}=\sum_{t=1}^{k} c_{t}
$$

Доказательство. Докажем достаточность. Пусть выполняются равенства

$$
\sum_{i=1}^{m} a_{i}=\sum_{j=1}^{n} b_{j}=\sum_{t=1}^{k} c_{t}
$$

Тогда $M(a, b, c) \neq \varnothing$. Возьмем некоторую матрицу $x=\left\|x_{i j t}\right\| \in M(a, b, c)$ и по ее 
элементам построим матрицу $y=\left\|y_{i j t}\right\|_{(m+1) \times(n+1) \times(k+1)}$ согласно формуле

$$
y_{i j t}= \begin{cases}x_{i j t}, & \text { если }(i, j, t) \in N_{m} \times N_{n} \times N_{k}, \\ R-\sum_{s=1}^{m} x_{s j t}, & \text { если } i=m+1,(j, t) \in N_{n} \times N_{k}, \\ R-\sum_{s=1}^{n} x_{i s t}, & \text { если } j=n+1,(i, t) \in N_{m} \times N_{k}, \\ R-\sum_{s=1}^{k} x_{i j s}, & \text { если } t=k+1,(i, j) \in N_{m} \times N_{n}, \\ 0, & \text { если }(i, j, t) \in G \\ R, & \text { если } i=m+1, j=n+1, t=k+1 .\end{cases}
$$

Непосредственной проверкой легко убедиться, что построенная по формуле (9) матрица $y \in M_{G}\left(A^{\prime}, B^{\prime}, C^{\prime}\right)$. Достаточность в утверждении леммы 4 доказана.

Докажем необходимость. Возьмем некоторую матрицу $y=\left\|y_{i j t}\right\| \in M_{G}\left(A^{\prime}, B^{\prime}, C^{\prime}\right)$ и по ее элементам построим матрицу $x=\left\|x_{i j t}\right\|_{m \times n \times k}$ соласно формуле

$$
x_{i j t}=y_{i j t} \quad \forall(i, j, t) \in N_{m} \times N_{n} \times N_{k} .
$$

Нетрудно проверить, что определенная таким образом матрица $x$ принадлежит многограннику $M(a, b, c)$. Лемма 4 доказана.

Лемма 5. Справедливо равенство

$$
\operatorname{dim} M_{G}\left(A^{\prime}, B^{\prime}, C^{\prime}\right)=m n k-m-n-k+2 .
$$

Доказательство. Повторяя рассуждения, приведенные в доказательстве леммы 3, получим равенство

$$
\operatorname{dim} M_{G}\left(A^{\prime}, B^{\prime}, C^{\prime}\right)=\operatorname{dim} M(a, b, c) .
$$

Теперь, учитывая, что $\operatorname{dim} M(a, b, c)=m n k-m-n-k+2$ (см. [1]), убеждаемся в справедливости леммы 5 .

Пусть задано отображение $\theta: M(a, b, c) \rightarrow M_{G}\left(A^{\prime}, B^{\prime}, C^{\prime}\right)$, которое каждой матрице $x \in M(a, b, c)$ по формуле (9) ставит в соответствие матрицу $y \in M_{G}\left(A^{\prime}, B^{\prime}, C^{\prime}\right)$.

Теорема 3. Отображение $\theta: M(a, b, c) \rightarrow M_{G}\left(A^{\prime}, B^{\prime}, C^{\prime}\right)$ обладает следующими свойствами:

(1) $\theta$ - взаимно однозначное отображение, имеющее обратное отображение $\theta^{-1}: M_{G}\left(A^{\prime}, B^{\prime}, C^{\prime}\right) . \rightarrow M(a, b, c)$, определяемое формулой $(10) ;$

(2) точка $x$ является верииной многогранника $M(a, b, c)$ тогда и толъко тогда, когда $\theta(x)$ - вершина многогранника $M_{G}\left(A^{\prime}, B^{\prime}, C^{\prime}\right)$;

(3) множество $F$ является гранъю многогранника $M(a, b, c)$ тогда и толъко тогда, когда $\theta(F)$ - грань многогранника $M_{G}\left(A^{\prime}, B^{\prime}, C^{\prime}\right)$.

Теорема 3 доказывается так же, как и теорема 1.

Из теоремы 3 вытекает следующее утверждение.

Следствие 4. Многогранник $M_{G}\left(A^{\prime}, B^{\prime}, C^{\prime}\right)$ порядка $(m+1) \times(n+1) \times(k+1)$ всегда является вирожденным. 
Теорема 4. Справедливо соотношение

$$
M(a, b, c) \cong M_{G}\left(A^{\prime}, B^{\prime}, C^{\prime}\right) .
$$

Доказательство теоремы 4 аналогично доказательству теоремы 2.

Отметим, что теорема 4 позволяет свести нахождение экстремума линейной функции на 3-АТМ $M(a, b, c)$ порядка $m \times n \times k$ к решению аналогичной задачи на многограннике $M_{G}\left(A^{\prime}, B^{\prime}, C^{\prime}\right)$ порядка $(m+1) \times(n+1) \times(k+1)$. На принципиальную возможность такого сведения ранее указывалось в [17].

На основании леммы 5 и теоремы 4 множество $M_{G}\left(A^{\prime}, B^{\prime}, C^{\prime}\right)$ есть планарное представление 3-АТМ $M(a, b, c)$.

Из теоремы 4 вытекает следующее утверждение.

Следствие 5. Граф 3-ATM $M(a, b, c)$ порядка $m \times n \times k$ изоморфен графу многогранника $M_{G}\left(A^{\prime}, B^{\prime}, C^{\prime}\right)$ порядка $(m+1) \times(n+1) \times(k+1)$.

На основании теоремы 4 и следствия 5 получаем следующие свойства:

(1) $\operatorname{dim} F=\operatorname{dim} \tau(F)$ и $F \cong \tau(F)$;

(2) $f_{s}(M(a, b ; c))=f_{s}\left(M_{G}\left(A^{\prime}, B^{\prime}, C^{\prime}\right)\right), \quad s=0,1,2, \ldots, m n k-m-n-k+1$;

(3) $\operatorname{diam} M(a, b, c)=\operatorname{diam} M_{G}\left(A^{\prime}, B^{\prime}, C^{\prime}\right)$;

(4) $r(M(a, b, c))=r\left(M_{G}\left(A^{\prime}, B^{\prime}, C^{\prime}\right)\right)$.

Здесь $\tau$ - отображение между множествами граней всех размерностей многогранников $M(a, b, c)$ и $M_{G}\left(A^{\prime}, B^{\prime}, C^{\prime}\right)$.

\section{4. Оценка снизу максимального числа вершин 3-ПТМ}

Для двух 3-ПТМ $M\left(A_{0}, B_{0}, C_{0}\right)$ и $M\left(A_{1}, B_{1}, C_{1}\right)$ одного и того же порядка рассмотрим матрицы:

$$
A_{\lambda}=\lambda A_{1}+(1-\lambda) A_{0}, \quad B_{\lambda}=\lambda B_{1}+(1-\lambda) B_{0}, \quad C_{\lambda}=\lambda C_{1}+(1-\lambda) C_{0},
$$

где $\lambda \in[0,1]$.

Спектром многогранников $M\left(A_{0}, B_{0}, C_{0}\right)$ и $M\left(A_{1}, B_{1}, C_{1}\right)$ назовем множество $S\left(A_{0}, B_{0}, C_{0}, A_{1}, B_{1}, C_{1}\right)$ всех чисел $\lambda \in(0,1)$, для каждого из которых $M\left(A_{\lambda}, B_{\lambda}, C_{\lambda}\right)$ - вырожденный многогранник.

Описание класса всех 3-ПТМ заданного порядка с максимальным числом вершин дает следующая теорема.

Теорема 5 ([10]). 3-ПTM $M(A, B, C)$ порядка $m \times n \times k, \quad m, n \geqslant 3, k \geqslant 2$, имеет максимальное число вериин тогда и только тогда, когда он невырожден и спектр $S\left(A, B, C, A^{*}, B^{*}, C^{*}\right)=\varnothing$, где

$$
A^{*}=\left[\begin{array}{ccc}
n & \ldots & n \\
n & \ldots & n \\
& \ldots & \\
n & \ldots & n
\end{array}\right] \quad B^{*}=\left[\begin{array}{ccc}
m & \ldots & m \\
m & \ldots & m \\
& \ldots & . \\
m & \ldots & m
\end{array}\right] \quad C^{*}=\left[\begin{array}{lll}
k & \ldots & k \\
k & \ldots & k \\
& \ldots & \\
k & \ldots & k
\end{array}\right] \text {. }
$$


Отыскание максимального числа $\varphi(m, n, k)$ вершин 3-ПТМ порядка $m \times n \times k$ представляет собой трудную задачу. Найти явную формулу даже для максимального числа $\varphi(n, k)$ вершин классического транспортного многогранника порядка $n \times k$ до сих пор не удалось. Известно [18], что

$$
\varphi(n, k) \geqslant k \varphi(n-1, k), \quad n, k \geqslant 2 .
$$

Следующая теорема дает рекуррентное неравенство, связывающее числа $\varphi(m, n, k)$ и $\varphi(m-1, n, k)$.

Теорема 6. Справедливо неравенство

$$
\varphi(m, n, k) \geqslant \varphi(n, k) \varphi(m-1, n, k) .
$$

Это утверждение в случае, когда $k=2$, впервые доказано в [19]. Там же была выведена формула

$$
\varphi(2, n, 2)=\varphi(n, 2)=\left(\begin{array}{c}
n \\
{[n / 2]}
\end{array}\right)(n-[n / 2]) .
$$

Доказательство теоремы 6. Пусть $M(A, B, C)-3$-ПТМ порядка $(m-1) \times n \times k$, для числа вершин которого справедливо равенство

$$
f_{0}(M(A, B, C))=\varphi(m-1, n, k) .
$$

Наряду с $M(A, B, C)$ рассмотрим 3-ПТМ $M\left(A^{\prime}, B^{\prime}, \mathrm{C}^{\prime}\right)$ порядка $m \times n \times k$, заданный матрицами $A^{\prime}, B^{\prime}$ и $C^{\prime}$, элементы которых определяются следующим образом:

$$
\begin{array}{rlrl}
a_{i t}^{\prime} & =a_{i t} & & \forall(i, t) \in N_{m-1} \times N_{k}, \\
a_{m t}^{\prime} & =\alpha n / k & & \forall t \in N_{k}, \\
b_{1 t}^{\prime} & =b_{1 t}+\frac{\alpha-(n-1) \beta}{k} & & \forall t \in N_{k}, \\
b_{j t}^{\prime} & =b_{j t}+\frac{\alpha+\beta}{k}, & j=2,3, \ldots, n & \forall t \in N_{k}, \\
c_{i j}^{\prime}=c_{i j} & & \forall(i, j) \in N_{m-1} \times N_{n}, \\
c_{m 1}^{\prime} & =\alpha-(n-1) \beta, & & \\
c_{m j}^{\prime} & =\alpha+\beta, & j=2,3, \ldots, n, &
\end{array}
$$

где $\alpha, \beta$ и $\beta / \alpha$ - сколь угодно малые положительные числа.

Пусть $M(c, a)$ - классический транспортный многогранник порядка $n \times k$, определенный векторами

$$
c=(\alpha-(n-1) \beta, \alpha+\beta, \ldots, \alpha+\beta), \quad a=(\alpha n / k, \ldots, \alpha n / k)
$$

размера $n$ и $k$ соответственно.

Прежде всего заметим, что

$$
M(A, B(y), C) \neq \varnothing \quad \forall y \in \operatorname{vert} M(c, a)
$$

где $B(y)=B^{\prime}-y$. 
Зафиксируем какую-нибудь вершину $y=\left\|y_{j t}\right\|_{n \times k} \in \operatorname{vert} M(c, a)$. Пусть $x=$ $\left\|x_{i j t}\right\|_{(m-1) \times n \times k}$ - произвольная вершина многогранника $M(A, B(y), C)$. Тогда, очевидно, матрица $z=\left\|z_{i j t}\right\|_{m \times n \times k}$ с элементами

$$
z_{i j t}= \begin{cases}x_{i j t}, & \text { если } i \in N_{m-1}, j \in N_{n}, t \in N_{k}, \\ y_{j t}, & \text { если } i=m, j \in N_{n}, t \in N_{k}\end{cases}
$$

является вершиной многогранника $M\left(A^{\prime}, B^{\prime}, C^{\prime}\right)$. Поэтому справедливо неравенство

$$
f_{0}\left(M\left(A^{\prime}, B^{\prime}, C^{\prime}\right)\right) \geqslant \sum_{y \in \operatorname{vert} M(c, a)} f_{0}(M(A, B(y), C)) .
$$

Поскольку $\alpha, \beta$ и $\beta / \alpha$ - сколь угодно малые положительные числа, выполняются равенства

$$
S(A, B, C, A, B(y), C)=\varnothing \quad \forall y \in \operatorname{vert} M(c, a) .
$$

Отсюда, принимая во внимание равенство (11), получаем соотношения

$$
S\left(A, B(y), C, A^{*}, B^{*}, C^{*}\right)=\varnothing \quad \forall y \in \operatorname{vert} M(c, a)
$$

Поэтому согласно теореме 5

$$
f_{0}(M(A, B(y), C))=\varphi(m-1, n, k) \quad \forall y \in \operatorname{vert} M(c, a) .
$$

Теперь, подставляя (13) в (12) и учитывая равенство $f_{0}(M(c, a))=\varphi(n, k)$ (см. теорему 7.1 в [1]), убеждаемся в справедливости теоремы 6.

Так как $\varphi(1, n, k)=\varphi(n, k)$, из теоремы 6 вытекает следующее утверждение.

Следствие 6. Справедливо неравенство

$$
\varphi(m, n, k) \geqslant \max \left\{(\varphi(n, k))^{m},(\varphi(m, k))^{n},(\varphi(m, n))^{k}\right\} .
$$

Из теоремы 2 и следствия 6 получаем следующее утверждение.

Следствие 7. Среди 3-ATM порядка $m k \times n k \times m n$ имеются многогранники, у которых найдется $(m-1)(n-1)(k-1)$-грань, содержащая по крайней мере $(\varphi(n, k))^{m}$ вериин.

\section{5. Оценка снизу максимального числа целочисленных вершин 3-ПТМ}

Так как всякий 3-ПТМ порядка $m \times n \times 2$, определенный целочисленными матрицами, является целочисленным, то есть все его вершины целочисленные [1], на основании теоремы 2 получаем следующий результат: среди 3 -АТМ порядка $2 m \times 2 n \times m n$ существуют многогранники, у которых найдется целочисленная $(m-1)(n-1)$-грань.

Через $f_{0}^{z}(M(A, B, C))$ будем обозначать число целочисленных вершин 3 -ПТМ $M(A, B, C)$, определенного целочисленными матрицами $A, B$ и $C$.

Теорема 7. Максимальное число целочисленных вершин в классе 3-ПТМ порядка $m \times n \times n$ не меньше $(n !)^{m-1}$. 
Доказательство. Рассмотрим 3-ПТМ $M(A, B, C)$ порядка $m \times n \times n$, определенный матрицами

$$
\begin{aligned}
& A=C=\left[\begin{array}{cccc}
(n-1) m+1 & (n-1) m+1 & \ldots & (n-1) m+1 \\
1 & 1 & \ldots & 1 \\
1 & 1 & \ldots & 1
\end{array}\right] \\
& B=\left[\begin{array}{llll}
m & m & \ldots & m \\
m & m & \ldots & m \\
& & \ldots & \\
m & m & \ldots & m
\end{array}\right]
\end{aligned}
$$

Наряду с многогранником $M(A, B, C)$ рассмотрим классические транспортные многогранники $M\left(a^{t}, b^{t}\right), t \in N_{n}$, порядка $m \times n$, каждый из которых определен векторами

$$
a^{t}=(m(n-1)+1,1,1, \ldots, 1), \quad b^{t}=(m, m, \ldots, m) .
$$

Для любого числа $t \in N_{n}$ и любой вершины $x^{t}=\left\|x_{i j}^{t}\right\|_{m \times n}$ многогранника $M\left(a^{t}, b^{t}\right)$ справедливы неравенства $x_{1 j}^{t}>0, j \in N_{n}$. Следовательно, так как число положительных компонент вершины $x^{t}$ не превосходит $m+n-1$, в строке с любым номером $i, i=2, \ldots, m$, имеется только одна положительная компонента (равная единице), и она может находиться в любом столбце. Номер этого столбца будем обозначать через $j_{i}^{t}$.

Пусть для любых двух чисел $t_{1}, t_{2} \in N_{n}, t_{1} \neq t_{2}$, имеет место соотношение

$$
\left\{\left(2, j_{2}^{t_{1}}\right),\left(3, j_{3}^{t_{1}}\right), \ldots,\left(m, j_{m}^{t_{1}}\right)\right\} \cap\left\{\left(2, j_{2}^{t_{2}}\right),\left(3, j_{3}^{t_{2}}\right), \ldots,\left(m, j_{m}^{t_{2}}\right)\right\}=\varnothing .
$$

Отсюда с учетом равенств

$$
c_{i j}=1, \quad i=2,3, \ldots, m, \quad \forall j \in N_{n},
$$

заключаем, что матрица $x=\left\|x_{i j t}\right\|_{m \times n \times n}$ с компонентами

$$
\begin{aligned}
& x_{i j t}= \begin{cases}1, & \text { если } j=j_{i}^{t}, \\
0, & \text { если } j \neq j_{i}^{t}, i=2, \ldots, m, \forall t \in N_{n},\end{cases} \\
& x_{1 j t}=m-\sum_{i=2}^{m} x_{i j t} \quad \forall j \in N_{n}, \quad \forall t \in N_{n},
\end{aligned}
$$

является целочисленной вершиной 3 -ПТМ $M(A, B, C)$. Поскольку число $(m-1)$ выборок $\left\{j_{2}^{t}, j_{3}^{t}, \ldots, j_{m}^{t}\right\}, t \in N_{n}$, для которых выполняется условие (14), равно $\prod_{t=1}^{n}(n-t+1)^{m-1}$, легко убедиться в том, что

$$
f_{0}^{z}(M(A, B, C)) \geqslant \prod_{t=1}^{n}(n-t+1)^{m-1}=(n !)^{m-1} \text {. }
$$

Теорема 7 доказана.

Из теорем 2 и 7 вытекает следующее утверждение.

Следствие 8. Среди 3-AТM порядка $m n \times n^{2} \times m n$, заданных целочисленными векторами, имеются многогранники, у которых найдется $(m-1)(n-1)(n-1)$-гранъ, содержащая по менъшей мере $(n !)^{m-1}$ целочисленных вершин. 


\section{6. Теорема о существовании 3-ПТМ, не содержащего целочисленных точек}

Теорема 8. В классе 3-ПТМ порядка $m \times n \times k, m \geqslant 6, n \geqslant 4, k \geqslant 3$, определенных целочисленными матрицами, имеются многогранники, у которых каждая точка является нецелочисленной.

Доказательство. Расссмотрим 3-ПТМ $M(A, B, C)$ порядка $m \times n \times k, m \geqslant 6, n \geqslant 4$, $k \geqslant 3$, определенный матрицами

$$
A=\left[\begin{array}{cc}
A_{1} & 0 \\
0 & A_{2}
\end{array}\right], \quad B=\left[\begin{array}{cc}
B_{1} & 0 \\
0 & B_{2}
\end{array}\right], \quad C=\left[\begin{array}{cc}
C_{1} & 0 \\
0 & C_{2}
\end{array}\right],
$$

где

$$
\begin{gathered}
A_{1}=\left[\begin{array}{lll}
1 & 0 & 1 \\
1 & 0 & 1 \\
0 & 1 & 1 \\
0 & 1 & 1 \\
1 & 1 & 0 \\
1 & 1 & 0
\end{array}\right], \quad B_{1}=\left[\begin{array}{lll}
1 & 1 & 1 \\
1 & 1 & 1 \\
1 & 1 & 1 \\
1 & 1 & 1
\end{array}\right], \quad C_{1}=\left[\begin{array}{llll}
0 & 1 & 0 & 1 \\
1 & 0 & 1 & 0 \\
1 & 0 & 0 & 1 \\
0 & 1 & 1 & 0 \\
1 & 1 & 0 & 0 \\
0 & 0 & 1 & 1
\end{array}\right], \\
A_{2}=\left[\begin{array}{llllll}
n-4 & n-4 & \ldots & n-4 \\
n-4 & n-4 & \ldots & n-4 \\
n-4 & n-4 & \ldots & n-4
\end{array}\right], \quad B_{2}=\left[\begin{array}{lllll}
m-6 & m-6 & \ldots & m-6 \\
m-6 & m-6 & \ldots & m-6 \\
m-6 & m-6 & \ldots & m-6
\end{array}\right], \\
C_{2}=\left[\begin{array}{lllll}
k-3 & k-3 & \ldots & k-3 \\
k-3 & k-3 & \ldots & k-3 \\
k-3 & k-3 & \ldots & k-3
\end{array}\right] .
\end{gathered}
$$

Нетрудно проверить, что нецелочисленная точка $x=\left\|x_{i j t}\right\|_{m \times n \times k}$, где

$$
\begin{array}{rlrl}
\left\|x_{i j t}\right\|_{m \times n} & =\left[\begin{array}{cc}
x^{t} & 0 \\
0 & 0
\end{array}\right], & t=1,2,3, \\
\left\|x_{i j t}\right\|_{m \times n}=\left[\begin{array}{cc}
0 & 0 \\
0 & x^{*}
\end{array}\right], & t=4,5, \ldots, k,
\end{array}
$$

где

$$
\begin{aligned}
& x^{1}=\left[\begin{array}{cccc}
0 & 1 / 2 & 0 & 1 / 2 \\
1 / 2 & 0 & 1 / 2 & 0 \\
0 & 0 & 0 & 0 \\
0 & 0 & 0 & 0 \\
1 / 2 & 1 / 2 & 0 & 0 \\
0 & 0 & 1 / 2 & 1 / 2
\end{array}\right], \quad x^{2}=\left[\begin{array}{cccc}
0 & 0 & 0 & 0 \\
0 & 0 & 0 & 0 \\
1 / 2 & 0 & 0 & 1 / 2 \\
0 & 1 / 2 & 1 / 2 & 0 \\
1 / 2 & 1 / 2 & 0 & 0 \\
0 & 0 & 1 / 2 & 1 / 2
\end{array}\right] \\
& x^{3}=\left[\begin{array}{cccc}
0 & 1 / 2 & 0 & 1 / 2 \\
1 / 2 & 0 & 1 / 2 & 0 \\
1 / 2 & 0 & 0 & 1 / 2 \\
0 & 1 / 2 & 1 / 2 & 0 \\
0 & 0 & 0 & 0 \\
0 & 0 & 0 & 0
\end{array}\right], \quad x^{*}=\left[\begin{array}{cccc}
1 & 1 & \ldots & 1 \\
1 & 1 & \ldots & 1 \\
& & \ldots & \\
1 & 1 & \ldots & 1
\end{array}\right]
\end{aligned}
$$


принадлежит многограннику $M(A, B, C)$.

Так как для любой точки $x=\left\|x_{i j t}\right\|_{m \times n \times k}$ многогранника $M(A, B, C)$ справедливы равенства

$$
\begin{aligned}
& x_{i j t}=0 \quad \forall i \in N_{6}, \quad j=5,6, \ldots, n, \quad \forall t \in N_{k}, \\
& x_{i j t}=0, \quad i=7,8, \ldots, m, \quad j=1,2,3,4, \quad t \in N_{k}, \\
& x_{i j t}=0 \quad \forall i \in N_{6}, \quad j=1,2,3,4, \quad t=4,5, \ldots, k \text {, } \\
& x_{i j t}=0 \quad i=7,8, \ldots, m, \quad j=5,6, \ldots, n, \quad t=1,2,3,
\end{aligned}
$$

многогранник $M(A, B, C)$ представим единственным образом в виде произведения многогранников $M\left(A_{1}, B_{1}, C_{1}\right), M\left(A_{2}, B_{2}, C_{2}\right)$ и четырех точек $P_{1}, P_{2}, P_{3}, P_{4}$ :

$$
M(A, B, C)=M\left(A_{1}, B_{1}, C_{1}\right) \otimes M\left(A_{2}, B_{2}, C_{2}\right) \otimes P_{1} \otimes P_{2} \otimes P_{3} \otimes P_{4},
$$

где $P_{1}, P_{2}, P_{3}, P_{4}$ - точки с координатами $(15),(16),(17),(18)$ соответственно. Поэтому для существования целочисленной точки в многограннике $M(A, B, C)$ необходимо и достаточно, чтобы многогранники $M\left(A_{1}, B_{1}, C_{1}\right)$ и $M\left(A_{2}, B_{2}, C_{2}\right)$ имели целочисленные точки. Отсюда получаем утверждение нашей теоремы, поскольку известно [20], что многогранник $M\left(A_{1}, B_{1}, C_{1}\right)$ не имеет ни одной целочисленной точки. Теорема 8 доказана.

Так как многогранник $M\left(A_{1}, B_{1}, C_{1}\right)$ вырождается в точку, на основании теорем 2 и 8 получаем следующее утверждение.

Следствие 9. Среди 3-.ATM порлдка $m k \times n k \times m n, m \geqslant 6, n \geqslant 4, k \geqslant 3$, заданных целочисленными векторами, имеются многогранники, среди граней которых найдется $(m-7)(n-5)(k-4)$-грань, все вершины которой являются нецелочисленными.

\section{7. Диаметр и радиус 3-ПТМ}

Известно [6], что всякое целое число вида $n+k-2+t$, где $t \in\{0,1, \ldots, n\}$, реализуется и как диаметр, и как радиус некоторого 3-АТМ порядка $m \times n \times k, m \geqslant n \geqslant k, m \geqslant 4$, $n \geqslant 3$. Поэтому на основании теоремы 4 получаем следующий результат.

Теорема 9. Всякое челое число вида $n+k-2+t$, где $t \in\{0,1, \ldots, n\}$, реализуется u как диаметр, и как радиус некоторого 3-ПТМ порядка $(m+1) \times(n+1) \times(k+1)$, $m \geqslant n \geqslant k, m \geqslant 4, n \geqslant 3$.

В [1] показано, что всякое целое число $\gamma, m n k-n k+n+k-1 \leqslant \gamma \leqslant m n k$, может быть числом фасет (граней максимальной размерности) некоторого 3-ПТМ порядка $m \times n \times k, m=\max (m, n, k)$.

Следствием теорем 4 и 4 из [6] является следующее утверждение.

Теорема 10. Пусть $m \geqslant 4, n \geqslant 3$. Тогда

- максималъный диаметр, так же, как и максимальный радиус, в классе 3ПТМ порядка $(m+1) \times(n+1) \times(k+1)$ с тnk $-n+t$ фасетами не менъше $n+k-2+t$, ecлu $t=0,1, \ldots, m-2, m$;

- максимальный диаметр в классе 3 -ПТМ порядка $(m+1) \times(n+1) \times(k+1)$ c mnk - 1 басетами не менъше $m+n+k-3$, а максималъный радиус в том же классе не менъие $m+n+k-4$. 


\section{Список литературы}

1. Емеличев В. А., Ковалев М. М., Кравцов М. К., Многогранники, графы, оптимизация. Наука, Москва, 1981.

2. Раскин Л. Г., Кириченко И. О., Многоиндексные задачи линейного программирования. Радио и связь, Москва, 1982.

3. Емеличев В. А., Кравцов М. К., Полиэдральные аспекты многоиндексных аксиальных транспортных задач. Дискретная математика (1991) 3, №2, 3-24.

4. Емеличев В. А., Кравцов М. К., Крачковский А. П., Многоиндексные планарные транспортные многогранники с максимальным числом вершин. Дискретная математика (1992) 4, №1, 3-18.

5. Bolker E. D., Simplicial geometry and transportation polytopes. Trans. Amer. Math. Soc. (1976) 217, 121-142.

6. Кравцов М. К., Полиэдральные аспекты многоиндексных транспортных задач с аксиальными суммами. Докл. АН СССР (1990) 315, №6, 1298-1302.

7. Емеличев В. А., Кравцов М. К., О некоторых свойствах транспортных многогранников с максимальным числом вершин. Докл. АН CCCP (1976) 228, №5, 1025-1028.

8. Емеличев В. А., Кравцов М. К., Крачковский А. П., О некоторых классах транспортных многогранников. Докл. АН ССCP (1978) 241, №3, 532-535.

9. Кравцов М. К., Диаметр и радиус транспортного многогранника. Докл. АН CCСP (1983) 270, №2, 278-281.

10. Емеличев В. А., Кравцов М. К., Крачковский А. П., Транспортные многогранники с максимальным числом $k$-граней. Докл. АН CССР (1985) 282, №4, 784-788.

11. Кравцов М. К., Полиэдральные аспекты транспортных задач. Докл. АН CCCP (1989) 309, №2, 271-275.

12. Even S., Itai A., Shamir A., On the complexity of timetable and multicommodity flow problems. SIAM J. Comput. (1976) 5, №4, 691-703.

13. Balas E., Saltzman M.J., Facets of the three-index assignment polytope. Discrete Appl. Math. (1989) 23, №3, 201-229.

14. Frieze A. M., Complexity of a 3-dimensional assignment problem. Europ. J. Oper. Research (1983) №13, 161-164.

15. Korsnikov A. D., Burkard R. E., On the dimension of polytopes of planar three-index. Optimization (1989) 20, №1, 107-116.

16. Емеличев В. А., Ковалев М. М., Полиэдральные аспекты дискретной оптимизации. Кибернетика (1982) №6, 54-62.

17. Haley K., The multi-index problem. Operat. Res. (1963) 11, №3, 369-379.

18. Klee V., Witzgall C., Facets and vertices of transportation polytopes. Proc. Amer. Math. Soc. (1968), $257-282$.

19. Кравцов М. К., Усеченные транспортные многогранники с максимальным числом вершин. Вести АН Беларуси. Сер. физ.-матем. наук (1993) №3, 30-34.

20. Vlach M., Conditions for the existence of solutions of the three-dimensional planar transportation problem. Discrete Appl. Math. (1986) 13, 61-78. 